\title{
Beta cell extracellular vesicle miR-21-5p cargo is increased in response to inflammatory cytokines and serves as a biomarker of type 1 diabetes
}

\author{
Alexander J. Lakhter ${ }^{1,2,3} \cdot$ Rachel E. Pratt ${ }^{1,2,3} \cdot$ Rachel E. Moore ${ }^{1,2,3} \cdot$ Kaitlin K. Doucette ${ }^{1,2,3} \cdot$ Bernhard F. Maier ${ }^{1,2}$. \\ Linda A. DiMeglio ${ }^{1,2,3}$. Emily K. Sims ${ }^{1,2,3}$
}

Received: 21 September 2017 / Accepted: 9 January 2018 / Published online: 14 February 2018

(C) Springer-Verlag GmbH Germany, part of Springer Nature 2018

\begin{abstract}
Aims/hypothesis Improved biomarkers are acutely needed for the detection of developing type 1 diabetes, prior to critical loss of beta cell mass. We previously demonstrated that elevated beta cell microRNA 21-5p (miR-21-5p) in rodent and human models of type 1 diabetes increased beta cell apoptosis. We hypothesised that the inflammatory milieu of developing diabetes may also increase miR-21-5p in beta cell extracellular vesicle (EV) cargo and that circulating EV miR-21-5p would be increased during type 1 diabetes development.

Methods MIN6 and EndoC- $\beta$ H1 beta cell lines and human islets were treated with IL- $1 \beta$, IFN- $\gamma$ and TNF- $\alpha$ to mimic the inflammatory milieu of early type 1 diabetes. Serum was collected weekly from 8 -week-old female NOD mice until diabetes onset. Sera from a cross-section of 19 children at the time of type 1 diabetes diagnosis and 16 healthy children were also analysed. EVs were isolated from cell culture media or serum using sequential ultracentrifugation or ExoQuick precipitation and EV miRNAs were assayed.

Results Cytokine treatment in beta cell lines and human islets resulted in a 1.5- to threefold increase in miR-21-5p. However, corresponding EVs were further enriched for this miRNA, with a three- to sixfold EV miR-21-5p increase in response to cytokine treatment. This difference was only partially reduced by pre-treatment of beta cells with Z-VAD-FMK to inhibit cytokine-induced caspase activity. Nanoparticle tracking analysis showed cytokines to have no effect on the number of EVs, implicating specific changes within EV cargo as being responsible for the increase in beta cell EV miR-21-5p. Sequential ultracentrifugation to separate EVs by size suggested that this effect was mostly due to cytokine-induced increases in exosome miR-21-5p. Longitudinal serum collections from NOD mice showed that EVs displayed progressive increases in miR-21-5p beginning 3 weeks prior to diabetes onset. To validate the relevance to human diabetes, we assayed serum from children with new-onset type 1 diabetes compared with healthy children. While total serum miR-21-5p and total serum EVs were reduced in diabetic participants, serum EV miR-21-5p was increased threefold compared with non-diabetic individuals. By contrast, both serum and EV miR-375-5p were increased in parallel among diabetic participants.

Conclusions/interpretation We propose that circulating EV miR-21-5p may be a promising marker of developing type 1 diabetes. Additionally, our findings highlight that, for certain miRNAs, total circulating miRNA levels are distinct from circulating EV miRNA content.
\end{abstract}

Keywords Beta cell signal transduction · Cell lines $\cdot$ Human $\cdot$ Prediction and prevention of type 1 diabetes

Electronic supplementary material The online version of this article (https://doi.org/10.1007/s00125-018-4559-5) contains peer-reviewed but unedited supplementary material, which is available to authorised users.

Emily K. Sims

eksims@iu.edu

1 Department of Pediatrics, Section of Pediatric Endocrinology and Diabetology, Indiana University School of Medicine, Indianapolis, IN, USA
2 Center for Diabetes and Metabolic Diseases, Indiana University School of Medicine, 635 Barnhill Dr., Rm 2031, Indianapolis, IN 46202, USA

3 Wells Center for Pediatric Research, Indiana University School of Medicine, Indianapolis, IN, USA 


\section{Research in context}

\section{What is already known about this subject?}

- Extracellular vesicle (EV) contents change over the course of disease development

- miR-21-5p is increased in beta cells and mouse and human islets under proinflammatory conditions

- Elevated levels of miR-21-5p are detrimental to beta cell survival

\section{What is the key question?}

- Does the inflammatory milieu of type 1 diabetes increase miR-21-5p levels in pancreatic beta cell EVs, and could this increase be detected in circulation?

\section{What are the new findings?}

- Beta cell EV miR-21-5p cargo is increased in models of inflammatory stress

- Elevations in serum EV miR-21-5p precede the onset of diabetes in NOD mice

- $\quad$ Although total serum miR-21-5p is decreased, serum EV miR-21-5p is increased in individuals with new-onset type 1 diabetes. By contrast, miR-375 is increased in both total serum and in serum EVs in the same group

How might this impact on clinical practice in the foreseeable future?

- Robust biomarkers of beta cell health could ultimately allow improved detection of preclinical type 1 diabetes and earlier administration of prevention strategies

$\begin{array}{ll}\text { Abbreviations } \\ \text { EndoC } & \text { EndoC- } \beta \mathrm{H} 1 \\ \text { EV } & \text { Extracellular vesicle } \\ \text { miR-21-5p } & \text { microRNA 21-5p } \\ \text { NOR } & \text { Non-obese diabetes-resistant } \\ \text { NTA } & \text { Nanoparticle tracking analysis } \\ \text { qPCR } & \text { Quantitative real-time PCR } \\ \text { TEM } & \text { Transmission electron microscopy }\end{array}$

\section{Introduction}

The global incidence of type 1 diabetes has been consistently rising by $3-5 \%$ a year, affecting millions of people worldwide $[1,2]$. Type 1 diabetes develops over time, with progressive dysfunction and destruction of pancreatic beta cells, such that by the time of clinical disease presentation, affected individuals have lost a substantial portion of their functional beta cell mass [3, 4]. Emerging data have suggested that the beta cell itself may contribute to type 1 diabetes development via activation of intrinsic stress pathways that exacerbate or accelerate autoimmune mediated destruction $[5,6]$. These data highlight a need for robust biomarkers of beta cell health to identify and monitor beta cell dysfunction. Such strategies would not only improve our understanding of the contribution made by beta cell dysfunction to the natural history of type 1 diabetes but would also enable the detection of developing diabetes, permitting earlier administration of disease-modifying therapies.
Extracellular vesicles (EVs) are membrane-bound nanoparticles released from all cell types that function in intercellular communication $[7,8]$. EVs are often broadly classified into three categories, exosomes, microvesicles and apoptotic bodies, based on their size, cellular origin and formation mechanism [9]. Research continues to elucidate distinct biological functions among these groups $[10,11]$. The molecular cargo of EVs partially mirrors the cellular contents of their cells of origin but can also reflect regulated changes occurring in conditions of stress or disease [12,13]. Because of this, EVs have generated interest as non-invasive biomarkers of multiple pathologic processes $[14,15]$.

We and others have previously demonstrated that beta cell microRNA $21-5 p$ (miR-21-5p) is increased in response to treatment with inflammatory cytokines and in models of type 1 diabetes, and that miR-21-5p has a negative impact on beta cell survival and function [7, 16-18]. In this context, increased miR-21-5p appears to be specific to beta cell inflammation, as treatment with high glucose to mimic hyperglycaemia or tunicamycin to induce endoplasmic reticulum stress does not affect miR21-5p expression [7]. Several groups have reported that circulating levels of miR-21-5p are increased in longstanding type 1 diabetes $[19,20]$. However, the aetiology of these observed increases in circulating miR-21-5p, and miR-21-5p's potential as a biomarker of developing type 1 diabetes, remain unexplored. Based on our earlier work, we predicted that beta cell EV miR-21-5p would be increased by exposure to the microenvironment of developing type 1 diabetes and, as 
a result, that circulating EV miR-21-5p could function as a biomarker of developing type 1 diabetes. Here, we detail our findings using both in vitro and in vivo models, as well as comparing clinical samples taken from children at the time of type 1 diabetes diagnosis with samples taken from healthy non-diabetic children.

\section{Methods}

Culture of cells and human islets MIN6 cells, originally obtained from J. Miyazaki (Osaka University Graduate School of Medicine, Suita, Osaka, Japan), were cultured as described previously, but with the use of EV-depleted FBS (ThermoFisher, Waltham, MA, USA) [21]. EndoC- $\beta \mathrm{H} 1$ (EndoC) cells, originally obtained from R. Scharfmann (Research Center Growth and Signaling, Université Paris Descartes, Paris, France), were cultured in serum-free media, as previously described [22]. Human islets were obtained through the Integrated Islet Distribution Program, which is exempt from institutional review board approval, and cultured in Standard Islet Medium (Prodo Labs, Aliso Viejo, CA, USA) supplemented with Human AB Serum (Prodo), glutamine and glutathione (Prodo) and $10 \mu \mathrm{g} / \mathrm{ml}$ ciprofloxacin (Corning, Corning, NY, USA), and depleted of EVs by overnight ultracentrifugation. The authenticity of cell lines was verified through maintenance of glucose-stimulated insulin secretion. Cells were routinely tested for mycoplasma with QuickTest Mycoplasma Detection Kit (Biotool, Houston, TX, USA). Absence of EVs in the media prior to culture was verified by nanoparticle tracking analysis (NTA). To model the inflammatory milieu of type 1 diabetes, samples were exposed to cytokine mix consisting of $5 \mathrm{ng} / \mathrm{ml} \mathrm{IL}-1 \beta$, $100 \mathrm{ng} / \mathrm{ml} \mathrm{IFN}-\gamma$ and $10 \mathrm{ng} / \mathrm{ml} \mathrm{TNF}-\alpha$ (R\&D Systems, Minneapolis, MN, USA) for $24 \mathrm{~h}$, as described previously [7]. Available cells and islet preparations were not randomised but divided evenly for comparison of control and cytokine treatments among islets from the same donor or cells from the same parent cells. To inhibit cytokine-induced apoptosis, $25 \mu \mathrm{mol} / \mathrm{l}$ of the pan-caspase inhibitor Z-VAD-FMK (R\&D Systems) was added to the cells concurrently with cytokines. Data are representative of at least three independent experiments.

Isolation of EVs Total EVs were isolated from culture media using ExoQuick TC reagent (System Biosciences, Palo Alto, CA, USA) and circulating EVs were isolated from $50 \mu \mathrm{l}$ of serum using ExoQuick reagent (System Biosciences) following manufacturer's guidelines. Sequential ultracentrifugation was used to separate EVs by size. The samples were centrifuged at $800 \mathrm{~g}$ for $15 \mathrm{~min}$ to remove dead cells and cellular debris, after which the supernatant fraction was centrifuged at $2000 \mathrm{~g}$ to pellet large EVs/apoptotic bodies. To collect microvesicles, the supernatant fraction from the previous step was centrifuged at $10,000 \mathrm{~g}$ and the resulting supernatant fraction was centrifuged at $100,000 \mathrm{~g}$ to pellet the exosomes. The remaining EV-depleted supernatant was also retained for analysis. EVs collected at each step were washed in PBS following described protocols and collected by centrifugation at appropriate speed $[23,24]$. Isolation and relative purity of the EVs were confirmed by NTA, transmission electron microscopy (TEM) and immunoblot.

PCR RNA isolation and reverse transcription were performed using miRNeasy and miScript II RT kits (Qiagen, Valencia, CA, USA) according to the manufacturer's instructions. Where applicable, RNA integrity was determined with Agilent Small RNA kit using a Bioanalyzer instrument (Agilent Technologies, Santa Clara, CA, USA). Quantitative real-time PCR (qPCR) was performed using the SooFast EvaGreen Supermix (BioRad, Hercules, CA, USA) and a Mastercycler ep realplex instrument (Eppendorf, Happauge, NY, USA). Due to low concentrations, droplet digital PCR (ddPCR) was performed as previously described to quantify serum EV miR-21-3p [25]. Primer for RNU6-1 was purchased from Sigma (St. Louis, MO, USA) and primers for miR-213p, miR-375-5p and the C. elegans miR-39 spike-in control were purchased from Qiagen. The following primer sequence was used to amplify miR-21-5p: CTAGCTTATCAGAC TGATGTTG. Qiagen's universal primer was used as the reverse primer in all reactions. Relative miRNA levels from EV isolates were established against $C$. elegans miR-39 mimic spike-in control, and from cells and tissues relative to $R N U 6-1$, using the $\Delta \Delta \mathrm{Cq}$ method.

Immunoblot analysis Immunoblot analysis was performed as described previously [7]. Antibodies (diluted 1:1000) against calreticulin, CD9 (10292-1-AP and 20597-1-AP; ProteinTech, Rosemon, IL, USA) and CD63 (ABIN1440014; AntibodiesOnline, Atlanta, GA, USA) were used for primary detection. Fluorescently labelled secondary antibodies (Jackson ImmunoResearch, West Grove, PA, USA) were used at 1:20,000 dilution. Signal detection was conducted on a LICOR Odyssey imager (LI-COR Biosciences, Lincoln, NE, USA). The antibodies were selected based on published literature. For further validation, protein lysates were serially diluted and subjected to immunoblot analysis. Distinct band signal of appropriate molecular weight and of intensity proportional to sample dilutions were considered validated for target detection by immunoblot.

NTA Samples enriched for EVs of interest were analysed for concentration and size distribution with dynamic light scattering using the ZetaView instrument (ParticleMetrix, Meerbusch, Germany). All data acquisition was conducted at room temperature. NTA measurements were recorded and 
analysed at 11 positions per sample with ZetaView Analyse software (ParticleMetrix).

TEM imaging EV-enriched sample preparations were fixed in EM-grade fixative containing $2 \%$ (wt/vol.) glutaraldehyde and $2 \%(\mathrm{wt} / \mathrm{vol}$.) paraformaldehyde in $0.1 \mathrm{~mol} / \mathrm{l}$ buffered phosphate for at least 30 mins at $4{ }^{\circ} \mathrm{C}$. After fixation, samples were placed on 200 mesh silicon monoxide formvar-coated grids and stained with NanoVan (Nanoprobes, Yaphank, NY, USA). Images were taken on an FEI Technai G2 Spirit TEM microscope (FEI, Houston, TX, USA).

Animal studies Eight-week-old female NOD (NOD/ShiLtJ) mice and non-obese diabetes-resistant (NOR/LtJ; NOR) control mice (The Jackson Laboratory, Bar Harbor, MI, USA) were followed with weekly blood glucose measurements and serum collections until the onset of diabetes (defined as first of two consecutive blood glucose values $>4.4 \mathrm{mmol} / \mathrm{l}$ ) [26]. All mice were euglycaemic and tested naive at initial evaluation. Non-diabetic NOD mice were followed until 20 weeks of age to rule out development of diabetes. Terminal serum was collected and islets were isolated as previously described [27]. For islet isolation and terminal serum collection, a sample size of five NOR and four NOD mice were used. Sample sizes of $n=5$ for NOR control mice and $n=7-9$ (depending on time to diabetes) for NOD mice were chosen for longitudinal experiments because of anticipated variability in prediabetic mice. Blood collection for serum isolation and glucose measurements were done via tail vein nick. Blood glucose was measured using an AlphaTRAK glucometer (Abbott Laboratories, Abbott Park, IL, USA) following manufacturer's instructions. Serum samples were isolated using a Microvette CB 300 system for capillary blood collection (Sarstedt, Numbrecht, Germany). Pancreatic islets were isolated using collagenase digestion [27]. The mice were maintained within the Indiana University Laboratory Animal Resource Center under pathogen-free conditions, in accordance with the Guide for the Care and Use of Laboratory Animals. All mice were kept in a standard light-dark cycle with ad libitum access to chow and water. All protocols were approved by the Indiana University School of Medicine Institutional Animal Care and Use Committee.

Human studies This study was approved by the Indiana University Institutional Review Board. Informed consent was obtained from parents with assent from children as required. Random serum samples were obtained from 19 children diagnosed with type 1 diabetes by a paediatric endocrinologist within 3 days of clinical diagnosis. $\mathrm{HbA}_{1 \mathrm{c}}$ levels were measured at diagnosis using either the BayerA $1 \mathrm{cNow}$ system or the Bayer DCA 2000 (Bayer, Tarrytown, NY, USA). Samples were collected in serum separator tubes and serum was isolated by centrifugation and stored at $-80^{\circ} \mathrm{C}$. Exclusion criteria included diabetic ketoacidosis requiring an intensive care unit stay, diabetes other than type 1 diabetes, history of prior chronic illness known to affect glucose metabolism or use of medications known to affect glucose metabolism. Serum from 16 healthy non-diabetic children (as a control group) was obtained from a biorepository at Indiana University School of Medicine. All available samples from the type 1 diabetes and healthy control groups were assayed in this analysis. All samples were analysed simultaneously by blinded laboratory personnel.

Statistical analyses GraphPad Prism 7 (GraphPad Software, La Jolla, CA, USA) was used for statistical analyses. Significance was assessed by a two-tailed Student's $t$ test or Mann-Whitney $U$ test (for nonparametric distributions), oneway ANOVA with Holm-Sidak's multiple comparisons test or two-way ANOVA with Sidak's multiple comparisons test for analysis of more than two groups. Spearman's correlation analyses were used to measure monotonic relationships. $p$ values were considered statistically significant when $p<0.05$.

\section{Results}

Beta cell EV miR-21-5p release is increased after exposure to inflammatory cytokines We and others have reported increased beta cell miR-21-5p expression after treatment with an inflammatory cytokine mix to model the type 1 diabetes inflammatory milieu [7, 17]. Consistent with this, we observed a 1.5- to threefold increase in miR-21-5p expression in MIN6 mouse insulinoma beta cells, EndoC human beta cells and human islets after treatment for $24 \mathrm{~h}$ with IL- $1 \beta$, INF- $\gamma$ and TNF- $\alpha$ (Fig. 1a-c). To determine whether this model of proinflammatory cytokine stress increases beta cell EV miR-21-5p, we also quantified miR-21-5p in EVs from MIN6 cells, EndoC cells and human islets after treatment for $24 \mathrm{~h}$ with cytokine mix. Here, cytokine exposure induced a three- to sixfold increase in total EV miR-21-5p (Fig. 1d-f), suggesting a selective enrichment of EV miR-21-5p in response to cytokines, rather than a non-specific reflection of a cytokine-induced increase in intracellular miR-21-5p.

To understand whether this difference in EV miR-21-5p correlated with increased release of EVs under inflammatory conditions, we performed NTA to determine EV quantity and size distribution. No significant differences in either the size distribution or particle concentration were detected in MIN6 or EndoC cells, or in human islets after $24 \mathrm{~h}$ of cytokine treatment (Fig. 1g-i). These data suggest that the cytokineinduced increase in beta cell EV miR-21-5p is specifically due to increases in vesicle cargo.

We further wanted to discover whether the cytokineinduced increase in EV miR-21-5p was related to cytokineinduced beta cell apoptosis. We measured EV miR-21-5p in 

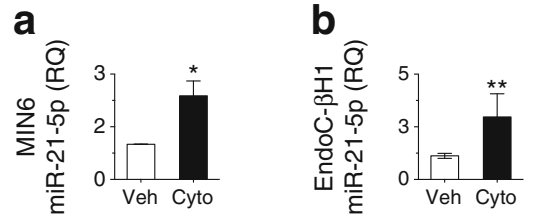

C
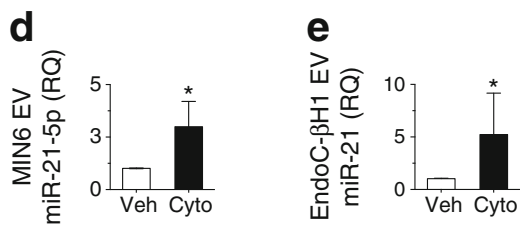

f

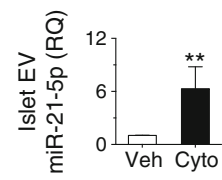

h
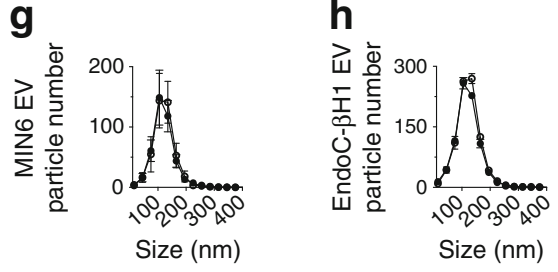

i

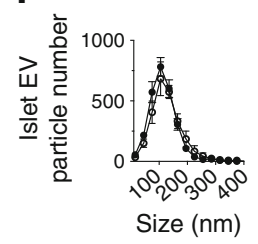

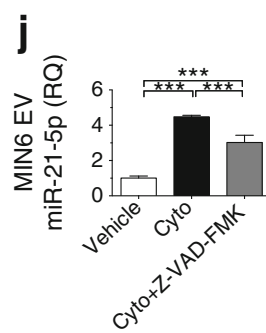

k

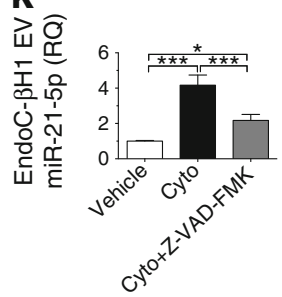

Fig. 1 Inflammatory cytokines increase beta cell EV miR-21-5p cargo. MIN6 and EndoC beta cells and human islets were treated with vehicle or a mix of IL-1 $\beta$, INF- $\gamma$ and TNF- $\alpha$ for $24 \mathrm{~h}$. (a-c) miR-21-5p expression was assessed in MIN6 cells $(n=3)(\mathbf{a})$, EndoC cells $(n=6)(\mathbf{b})$ and human islets $(n=10)(\mathbf{c}) .(\mathbf{d}-\mathbf{f}) \mathrm{miR}-21-5 \mathrm{p}$ levels were assessed in EVs from MIN6 cells $(n=3)(\mathbf{d})$, EndoC cells $(n=3)(\mathbf{e})$ and human islets $(n=6)(\mathbf{f})$. (g-i) NTA was performed to profile EV particle concentration and size distribution in MIN6 cells $(n=3)(\mathbf{g})$, EndoC beta cells $(n=3)(\mathbf{h})$ and human islets $(n=3)(\mathbf{i})$. (j, k) MIN6 cells $(n=3)(\mathbf{j})$ and EndoC cells $(n=3)(\mathbf{k})$ were treated with inflammatory cytokines or cytokines in combination with the pan-caspase inhibitor Z-VAD-FMK. Effects on relative levels of EV miR-21-5p were assessed by qPCR. Results are displayed as mean $\pm \mathrm{SD} ; * \leq \leq 0.05, * * \leq \leq 0.01$ and $* * * p \leq 0.001$. White circles, vehicle; black circles, cytokines. Cyto, cytokines; Veh, vehicle; RQ, relative quantity

MIN6 and EndoC cells treated with both cytokines and the pan-caspase inhibitor Z-VAD-FMK (Fig. 1k,1). Here, cytokine-induced increases in EV miR-21-5p were partially abrogated by addition of Z-VAD-FMK. However, EV miR$21-5 p$ levels remained approximately twofold increased compared with EVs from vehicle-treated cells, suggesting that the cytokine-induced increase in beta cell EV miR-21-5p is only partially related to beta cell death.

Cytokine-induced increase in beta cell EV miR-21-5p is predominantly due to beta cell exosome miR-21-5p Next, we asked whether the cytokine-induced increase in beta cell EV miR-21-5p was specific to a particular EV subtype. We used sequential ultracentrifugation to separate EVs by size,

allowing for enrichment for larger vesicles (apoptotic bodies), intermediate-sized vesicles (microvesicles) and smaller vesicles (exosomes) [10,28]. Immunoblot analysis and TEM were performed to validate isolations (Fig. 2a,b). Media remaining post-centrifugation was also collected to assess the presence and relative levels of EV-independent miR-21-5p release. miRNA quality and quantity were validated by spectral analysis, which revealed that miRNA concentrations in EV preparations from the vehicle- and cytokine-treated samples were similar (ESM Fig. 1). qPCR of each fraction revealed that in MIN6 cells, miR-21-5p was only increased by cytokines in the exosome fraction (Fig. 2c). In EndoC cells and human islets, a cytokine-induced increase in miR-21-5p occurred in the apoptotic body and exosome fractions (Fig. 2d,e). NTA of ultracentrifugation fractions revealed much higher total concentrations of exosomes than either microvesicles or apoptotic bodies (Fig. $2 \mathrm{f}-\mathrm{h}$ ). In aggregate, these data suggest that exosomes are the predominant type of vesicle contributing to the cytokine-induced increase in beta cell EV miR-21-5p.

Progressive elevation in circulating EV miR-21-5p precedes the onset of diabetes in NOD mice Based on the increased release of beta cell EV miR-21-5p in response to cytokine exposure ex vivo, we predicted that circulating EV miR-21$5 \mathrm{p}$ would also be increased during development of type 1 diabetes in vivo. To test this, we used the NOD mouse model of type 1 diabetes. Consistent with our data in cytokine-treated cells and human islets, islets from female diabetic NOD mice at the time of diabetes onset exhibited a 2.5 -fold increase in miR-21-5p expression compared with islets from NOR control mice (Fig. 3a). Next, we isolated EVs from terminal serum of diabetic mice and detected an approximate tenfold increase in serum EV miR-21-5p compared with NOR control mice (Fig. 3b). EV isolation was validated by western blotting for EV markers CD9 and CD63, which were enriched in the EV samples as compared with the original serum samples; as expected, the endoplasmic reticulum marker calreticulin was grossly depleted in the EV isolates (Fig. 3c).

To assess changes in circulating EV miR-21-5p during type 1 diabetes development, we collected weekly serial serum samples from NOD mice starting at 8 weeks of age until the time of diabetes onset (Fig. 3d, $n=7-9$ per timepoint depending on time of diabetes onset). We observed an age-dependent increase in miR-21-5p in the circulating EVs of prediabetic NOD mice starting at 12 weeks of age (Fig. 3e). To establish temporal relationships to diabetes development we normalised these values to age-matched NOR control mice and plotted the data according to relative time to diabetes onset. This revealed a progressive increase in EV miR-21-5p starting 3 weeks before development of hyperglycaemia (Fig. 3f). NTA of these circulating EVs revealed no significant changes in the EV concentration relative to either age or diabetes onset (Fig. $3 \mathrm{~g}, \mathrm{~h})$. These data confirmed that progressive increases in 
a

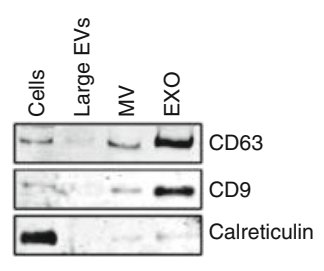

C

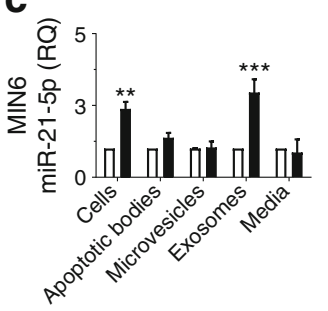

f

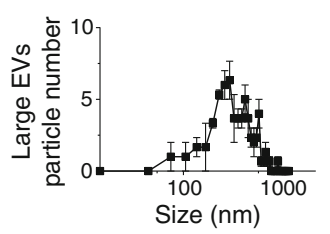

b
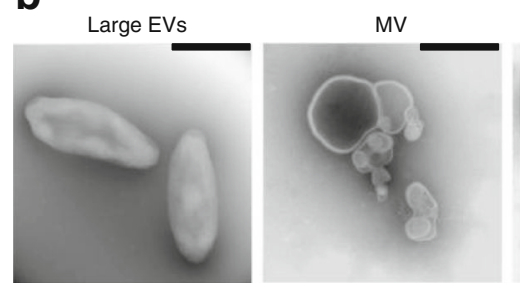

EXO

d

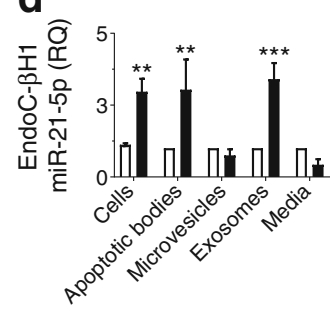

g

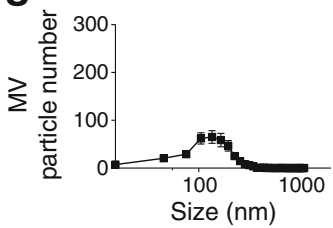

e

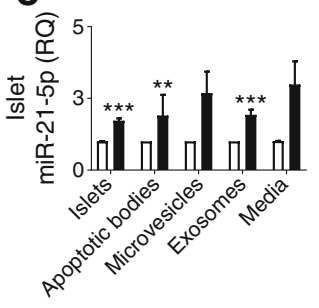

h

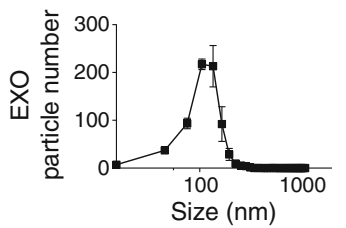

Fig. 2 Cytokine-induced increase in beta cell EV miR-21-5p is predominantly due to changes in exosome cargo. Media from vehicle- and cytokine-treated MIN6 cells, EndoC cells and human islets was separated by sequential centrifugation into large EV (apoptotic bodies), intermediate-sized EV (microvesicles) and small EV (exosomes) fractions. EVdepleted media was also retained. (a, b) Immunoblot (a) and TEM (b) analysis of EndoC cell-derived EVs was used to validate EV isolation by serial ultracentrifugation. TEM images display representative data from

serum EV miR-21-5p pre-date hyperglycaemia during developing diabetes in this mouse model of type 1 diabetes.

miR-21-5p is increased in circulating EVs from children with new-onset type 1 diabetes To establish relevance to human disease, we analysed clinical serum samples from 19 paediatric volunteers with new-onset type 1 diabetes and 16 healthy paediatric control participants. Demographic characteristics are presented in Table 1. No significant differences in age, sex or BMI percentile for age were present between the groups. We quantified total serum miR-21-5p, total serum EVs and circulating EV miR-21-5p. In contrast to reports in longstanding type 1 diabetes, total serum miR-21-5p was decreased in participants with recentonset type 1 diabetes compared with the healthy control group (Fig. 4a) [19, 20]. However, consistent with our in vitro and NOD mouse findings, levels of serum EV miR-21-5p were increased threefold in samples from children with type 1 diabetes (Fig. 4b). Furthermore, when normalised to the total serum miR21-5p level, the miR-21-5p levels from circulating EVs of participants with type 1 diabetes were fivefold higher than in control participants, confirming that the increase in serum miR-21-5p was specific to EVs (Fig. 4c). three independent experiments. Scale bars, $400 \mathrm{~nm}$. (c-e) Relative levels of miR-21-5p were assessed by qPCR. ( $n=3$ in MIN6 and EndoC cells; $n=5$ in human islets) (f-h) NTA of each ultracentrifugation fraction from EndoC cells was used to establish EV quantity and size distribution; $(n=3$ in MIN6 and EndoC cells; $n=5$ in human islets). White bars, vehicletreated media; black bars, cytokine-treated media. Results are displayed as mean $\pm \mathrm{SD} ; * * p \leq 0.01$ and $* * * p \leq 0.001$. EXO, exosomes; MV, microvesicles; RQ, relative quantity

NTA, performed on serum samples to assess differences in the overall concentration and size distribution of circulating EVs, revealed a reduced number of circulating EVs (particularly smaller EVs) in type 1 diabetes samples (Fig. $4 \mathrm{~d}-\mathrm{f}$ ). Normalisation of serum EV miR-21-5p to total particle count also revealed increased serum EV miR-21-5p in children with type 1 diabetes (Fig. 4g), consistent with our in vitro findings wherein increased EV miR-21-5p was related to increased EV miR-21-5p cargo rather than being reflective of increased EV release. We measured correlations between serum EV miR-21$5 p$ and age, BMI percentile and $\mathrm{HbA}_{1 \mathrm{c}}$ for diabetic and healthy participants. In the healthy individuals, younger age correlated with higher EV miR-21-5p levels $\left(r_{\mathrm{s}}=-0.506, p=0.0474\right.$; ESM Fig. 2a). However, this relationship was not present in participants with type 1 diabetes (ESM Fig. 2b). We did not detect any correlations between BMI percentile or $\mathrm{HbA}_{1 \mathrm{c}}$ and $\mathrm{EV}$ miR-21$5 p$ levels. Similarly, no differences were detected based on sex.

Given that miR-21-3p originates from the same precursor miRNA as miR-21-5p, we predicted that circulating EV miR21-3p may be increased in samples from participants with type 1 diabetes [29]. Consistent with prior reports in individuals at risk of type 1 diabetes [30], total serum miR-21-3p was 

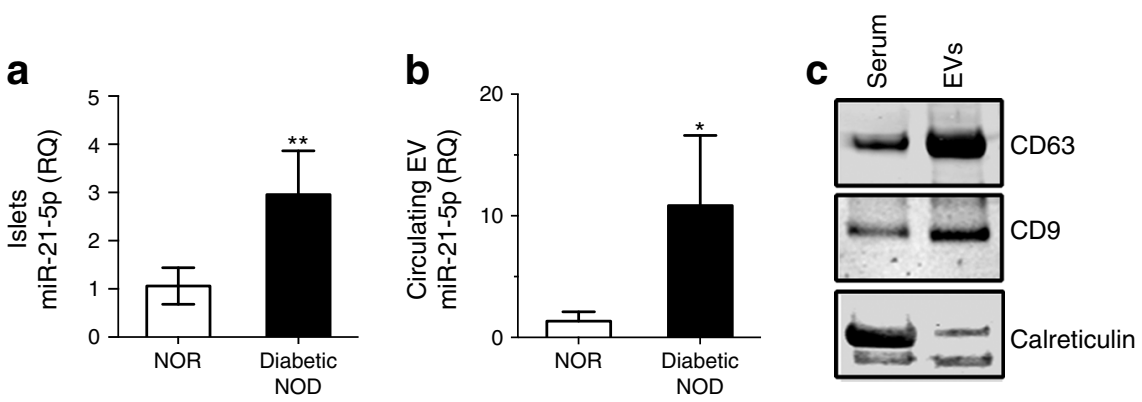
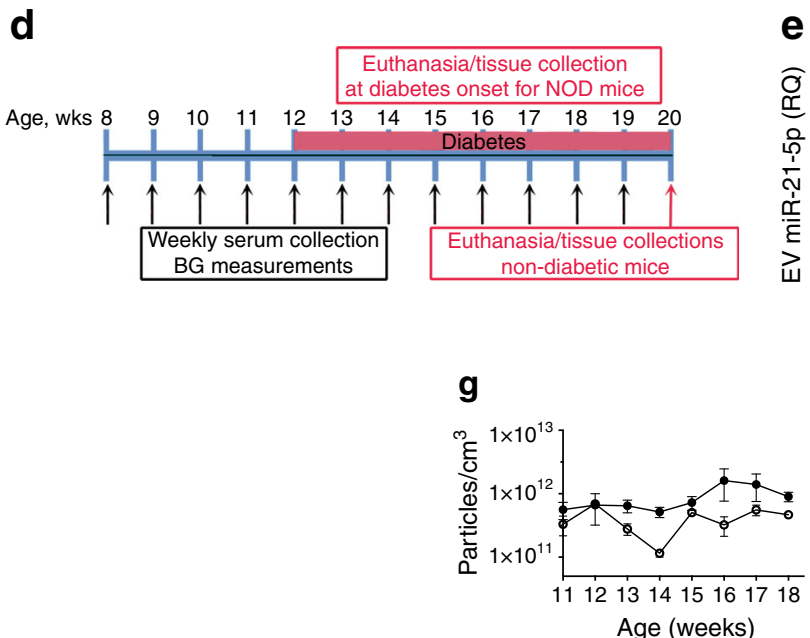

Fig. 3 Elevation in circulating EV miR-21-5p precedes onset of diabetes in NOD mice. (a, b) qPCR was performed to quantify relative levels of EV miR-21-5p in islets (a) and terminal serum (b) of diabetic NOD mice $(n=4)$ compared with NOR controls $(n=5)$. (c) Specificity of EV isolation was validated by western blotting of total serum and circulating EVs, probed for EV markers CD63 and CD9 and the endoplasmic reticulum marker calreticulin. (d) Longitudinal weekly serum collection and blood glucose assessment of NOD $(n=7-9)$ and control NOR $(n=5)$ mice were performed, starting at 8 weeks (wks) of age until either development of diabetes or 20 weeks of age. (e) qPCR was performed to quantify

increased in samples from participants with type 1 diabetes (ESM Fig. 2c). In contrast, median serum EV miR-21-3p levels were lower in samples from the type 1 diabetes group, despite there being considerable overlap in individual values between the two groups (ESM Fig. 2d).

We also asked whether our observed differences in serum and EV miRNAs would apply to a different miRNA

Table 1 Demographic characteristics of study participants

\begin{tabular}{llll}
\hline Variable & $\begin{array}{l}\text { Healthy controls } \\
(n=16)\end{array}$ & $\begin{array}{l}\text { Type 1 diabetes } \\
(n=19)\end{array}$ & $p$ value \\
\hline Age, years & $10.5(9-12)$ & $10.5(8-12)$ & 0.8579 \\
Male sex, \% & 62.5 & 62.5 & 1.00 \\
$\mathrm{BMI}$ percentile & $84(72.5-89.5)$ & $52.5(32.5-87.5)$ & 0.1343 \\
$\mathrm{HbA}_{1 \mathrm{c}}, \mathrm{mmol} / \mathrm{mol}$ & NA & $100(89-111)$ & NA \\
$\mathrm{HbA}_{1 \mathrm{c}}, \%$ & NA & $11.3(10.3-12.3)$ & NA \\
\hline
\end{tabular}

Results are displayed as median (interquartile range)
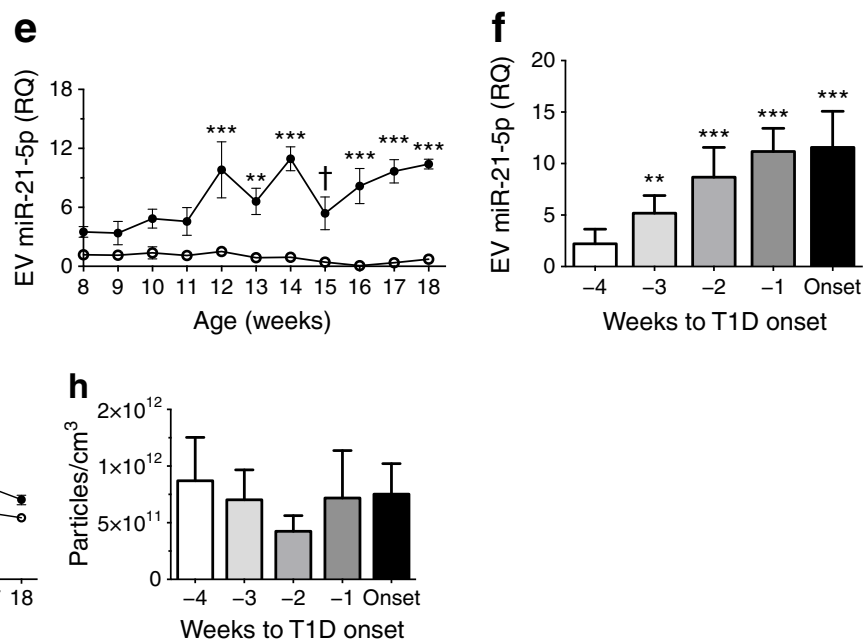

serum EV miR-21-5p in NOD mice vs NOR control mice according to age ( $n=3-9 /$ group). (f) Serum EV miR-21-5p in NOD mice and agematched NOR control mice was analysed in relation to diabetes onset (defined as first glucose $>4.4 \mathrm{mmol} / \mathrm{l}$ ) ( $n=3-9 /$ group). (g, h) NTA of serum EVs in NOD mice showed no significant changes with relation to age (g) or diabetes onset ( $n=3-9 /$ group) (h). Results are displayed as mean $\pm \mathrm{SD} ;{ }^{\dagger} p=0.053,{ }^{*} p \leq 0.05,{ }^{*} p \leq 0.01$ and ${ }^{* * *} p \leq 0.001$. White circles, NOR; black circles, NOD. BG, blood glucose; RQ, relative quantity, T1D, type 1 diabetes

biomarker of diabetes. We chose to measure miR-375-5p, which has been proposed as a circulating biomarker of beta cell death (Fig. 4h,i) [31, 32]. In contrast to miR-21-5p and miR-21-3p, in participants with type 1 diabetes, miR-375-5p was similarly increased in both serum and in serum EVs. We next examined associations between the relative levels of serum EV miR-21-5p and miR-375-5p among participants with diabetes. Although there was overlap in some individuals, the levels of the two microRNAs were not correlated overall, with some individuals displaying predominant elevations in either serum EV miR-21-5p or serum EV miR-375-5p (Fig. 4j).

\section{Discussion}

Our findings demonstrate that beta cell EV miR-21-5p cargo is increased in response to treatment with inflammatory cytokines. This increase was predominantly due to cytokine-induced effects on beta cell exosome miR-21-5p and was only partially blocked 

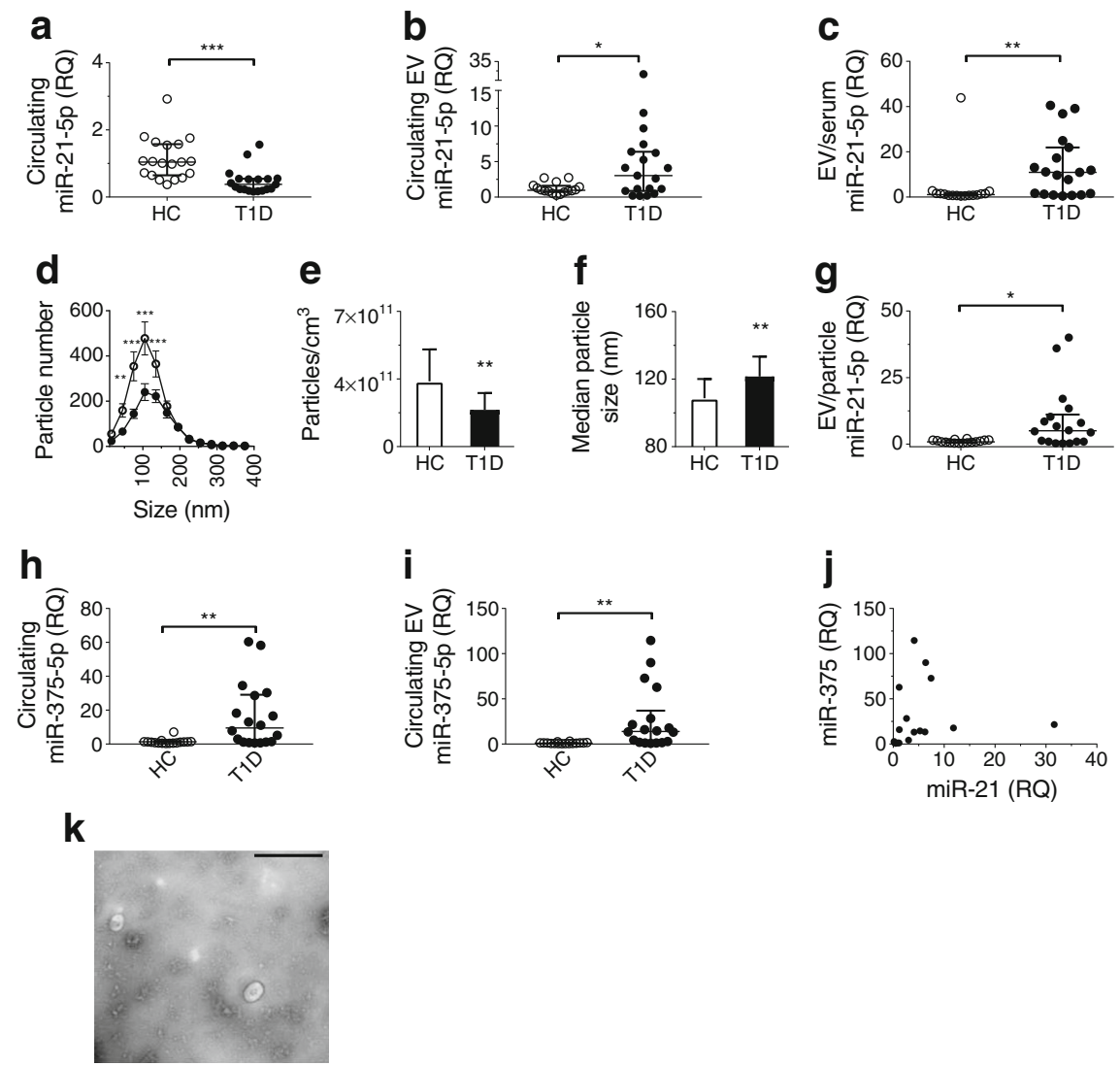

Fig. 4 Circulating EV miR-21-5p is elevated in children with newonset type 1 diabetes. (a, b) Serum samples from healthy control children and children with new-onset type 1 diabetes were assessed for relative levels of circulating miR-21-5p in whole serum (a) and in circulating EVs (b). (c) Circulating EV miR-21-5p normalised to total serum miR-21-5p. (d-f) NTA was performed to quantify serum EV concentration and size distribution. (e) Quantification of total particle concentration by group. (f) Quantification of particle size by group. (g) Serum EV miR-21-5p level normalised to total serum particle number. (h, i) Relative levels of total

by inhibition of apoptosis. We also report the novel observation that circulating EV miR-21-5p may be a biomarker of developing type 1 diabetes, in that progressive elevations in serum EV miR-21-5p preceded hyperglycaemia in NOD mice and were present in children with new-onset type 1 diabetes.

Prior miRNA profiling in beta cells has demonstrated that the exosome contents differ from the cytoplasmic contents of cells of origin [33]. Specifically, several reports have demonstrated that beta cell EVs and their cargo have physiological relevance to the type 1 diabetes microenvironment $[6,17$, 33-39]. Islet EVs contain beta cell autoantigens and are able to increase antigen presentation and inflammatory cytokine production by antigen-presenting cells, as well as stimulate endothelial cell angiogenesis [6, 34, 35, 39]. Incubation of human islets with circulating EVs from individuals with type 1 diabetes reduces glucose-stimulated insulin secretion [40]. Prior work has also shown that exosomes from cytokinetreated parent beta cells have a miRNA-dependent pro- serum miR-375-5p (h) and circulating EV miR-375-5p (i) were also quantified. (j) Relationship between serum EV miR-21-5p and EV miR-375-5p in samples from children with type 1 diabetes. (k) EV isolation from serum was validated by TEM; a representative image from three independent experiments is shown. Scale bar, $400 \mathrm{~nm}$. Results are shown as median \pm interquartile range $(\mathbf{a}-\mathbf{c}, \mathbf{e}-\mathbf{j})$ or mean $\pm \operatorname{SEM}(\mathbf{d}) ;{ }^{*} p \leq 0.05$, $* * p \leq 0.01$ and $* * * p \leq 0.001$. White circles, healthy controls $(n=16)$; black circles, type 1 diabetes $(n=19)$. HC, healthy controls; RQ, relative quantity; T1D, type 1 diabetes

apoptotic effect on recipient beta cells [33]. Our data point to an enrichment in the cytokine effect on miR-21-5p within beta cell exosomes, relative to intracellular miR-21-5p. Based on our previous report that miR-21-5p increases beta cell apoptosis, it is tempting to speculate that exosome miR-21-5p may be involved in the pro-apoptotic effect of exosomal miRNA transfer from cytokine-treated cells [7].

We observed clear differences between circulating total miR21-5p and EV miR-21-5p levels. Although long-term incubation of islets with cytokines has been reported to increase exosome release, we did not observe any change in beta cell or islet EV quantity after $24 \mathrm{~h}$ of cytokine treatment [6]. Lack of an increase in total EV count in islets or serum from individuals with type 1 diabetes suggests that our observed increases in EV miR-21-5p are related to increases in miR-21-5p cargo per EV. Our findings of parallel levels of miR-375-5p in total serum and serum EVs suggest that differential miRNA concentrations in serum vs serum EVs is not universal. These differences could reflect the 
pathophysiological process associated with release of the miRNA being studied [41]. For example, miR-375-5p may be non-selectively released by dying beta cells, as both EVindependent circulating miRNA and in EVs, while miRNAs reflecting other aspects of the diabetic islet microenvironment, such as miR-21-5p, could be more selectively packaged into EVs [41]. In aggregate, these findings confirm the importance of defining circulating EV miRNA content in addition to total circulating miRNA. Future work is needed to better understand the physiology of selective changes in beta cell EV miRNA cargo.

Our findings of decreased serum miR-21-5p in children with type 1 diabetes are in contrast to several published reports of increased circulating miR-21-5p in individuals with more established type 1 diabetes [19, 20]. However, reports describing total circulating miRNAs in new-onset type 1 diabetes or in those at risk of diabetes, which would be more clinically consistent with our study population, have not described elevated circulating miR-21-5p levels [30, 42, 43]. Although we did observe a clear subset of individuals with type 1 diabetes that displayed elevations in EV miR-21-5p, with half of our diabetes samples above the range for healthy volunteers, there was variation among individuals with type 1 diabetes as a group. Similarly, significant variation existed in serum EV miR-375-5p values among study participants with type 1 diabetes. This variation likely reflects the heterogeneous nature of type 1 diabetes, which has been observed in other clinical cohorts, and may also explain differences in reports of levels of circulating miRNAs from small cross-sectional studies [20, 25, 43-45]. Our finding that serum EV miR-21-5p and EV miR-375-5p levels were not necessarily correlated highlights the benefit of including multiple miRNAs as complementary tools to identify a signature of developing type 1 diabetes.

miR-21-5p is widely abundant, and released into circulation from multiple tissue sources. This contributes another likely source of variation to our study and significantly limits work to define the biomarker potential of circulating miRNAs in general [46]. However, our observations of consistent increases in EV miR-21-5p after cytokine exposure and before diabetes onset in the NOD mouse suggest that beta cell EV miR-21-5p is feasibly a major contributor to our observed signal in serum. Emerging strategies to isolate islet or beta cell-derived EVs from the circulation are likely to increase the specificity of this assay and allow for earlier and more precise detection of developing type 1 diabetes [38]. Alternatively, removal of EVs derived from other tissues or identification of exosome subpopulations responsible for increased exosome miR-21-5p may provide a more robust signal $[10,11,28]$.

In aggregate, our findings point to circulating EV miR-21$5 \mathrm{p}$ as a promising biomarker of developing type 1 diabetes. Future studies will define the pathophysiological significance of EV miR-21-5p transfer to recipient beta cells in the progression to type 1 diabetes and establish whether beta cell EV miR-21-5p transfer represents a feasible therapeutic target.
Studies are also needed to identify EV surface proteins that allow for enrichment of beta cell-specific EVs, and to define other beta cell EV miRNAs affected during the development of type 1 diabetes. Prospective evaluations of individuals are indicated to determine whether glycaemic control affects circulating EV miR-21-5p and whether levels are predictive of differences in type 1 diabetes clinical outcomes, such as honeymoon duration or loss of endogenous C-peptide secretion.

Acknowledgements We thank C. Evans-Molina and R. Mirmira (Departments of Medicine and Pediatrics, Indiana University School of Medicine) for their support in developing this project. We thank the Indiana University Islet and Physiology core for assistance with serum collection and islet isolation and the University of Nebraska College of Medicine Electron Microscopy Core for assistance with TEM imaging. This work has been partially presented in oral abstract form and in poster presentations at the 2015-2017 American Diabetes Association Scientific Sessions, 2017 Human Islet Research Network, Pediatric Endocrine Society, Endocrine Society, 2015-2016 Midwest Islet Club, 2016 Central Society for Clinical and Translational Research, 2017 Extracellular RNA Communication Consortium and the 2017 International Society for Extracellular Vesicles meeting.

Data availability The datasets generated during and/or analysed during the current study are available from the corresponding author on reasonable request.

Funding This work was supported by NIDDK K08DK103983 (EKS), Pediatric Endocrine Society Clinical Scholar Award (EKS), a pilot and feasibility award within the Center for Diabetes and Metabolic Diseases NIH/NIDDK grant number P30 DK097512, funding by Indiana University Health and the Indiana Clinical and Translational Sciences Institute (EKS), Grant no. UL1TR001108, a Ralph W. and Grace M. Showalter research award (EKS), JDRF Pioneer Award and Strategic Research Agreement (LAD) and NIH grant no. 32DK064466 (AJL). This study utilised core services provided by the Diabetes Research Center grant P30 DK097512 to Indiana University School of Medicine. Human pancreatic islets were provided by the NIDDK-funded Integrated Islet Distribution Program (IIDP) at City of Hope, NIH grant no. 2UC4DK098085-02.

Duality of interest The authors declare that there is no duality of interest associated with this manuscript.

Contribution statement AJL, REP, REM, and KKD performed the experiments, acquired the data and revised the manuscript. BFM made substantial contributions to study design and analysis of data and revised the manuscript. LAD made substantial contributions to acquisition of data and revised the manuscript. AJL and EKS designed the experiments, acquired and interpreted data, and drafted the manuscript. EKS is the guarantor of this work and, as such, had full access to all the data in the study and takes responsibility for the integrity of the data and the accuracy of the data analysis. All authors provided final approval of the version to be published.

\section{References}

1. Dabelea D, Mayer-Davis EJ, Saydah S et al (2014) Prevalence of type 1 and type 2 diabetes among children and adolescents from 2001 to 2009. JAMA 311:1778-1786

2. Maahs DM, West NA, Lawrence JM, Mayer-Davis EJ (2010) Chapter 1: epidemiology of type 1 diabetes. Endocrinol Metab Clin N Am 39:481-497 
3. Eisenbarth GS (1986) Type I diabetes mellitus. A chronic autoimmune disease. N Engl J Med 314:1360-1368

4. Atkinson MA, Eisenbarth GS, Michels AW (2014) Type 1 diabetes. Lancet 383:69-82

5. Heninger A-K, Eugster A, Kuehn D, et al. (2017) A divergent population of autoantigen-responsive $\mathrm{CD}^{+} \mathrm{T}$ cells in infants prior to $\beta$ cell autoimmunity. Science Translational Medicine 9

6. Cianciaruso C, Phelps EA, Pasquier M et al (2017) Primary human and rat beta-cells release the intracellular autoantigens GAD65, IA2 , and proinsulin in exosomes together with cytokine-induced enhancers of immunity. Diabetes 66:460-473

7. Sims EK, Lakhter AJ, Anderson-Baucum E, Kono T, Tong X, Evans-Molina C (2017) MicroRNA 21 targets BCL2 mRNA to increase apoptosis in rat and human beta cells. Diabetologia 60 : 1057-1065

8. Gould SJ, Raposo G (2013) As we wait: coping with an imperfect nomenclature for extracellular vesicles. J Extracell Vesicles. https:// doi.org/10.3402/jev.v2i0.20389

9. Yanez-Mo M, Siljander PR, Andreu Z et al (2015) Biological properties of extracellular vesicles and their physiological functions. J Extracell Vesicles 4:27066

10. Kowal J, Arras G, Colombo M et al (2016) Proteomic comparison defines novel markers to characterize heterogeneous populations of extracellular vesicle subtypes. Proc Natl Acad Sci U S A 113:E968E977

11. Willms E, Johansson HJ, Mäger I et al (2016) Cells release subpopulations of exosomes with distinct molecular and biological properties. Sci Rep 6:22519

12. Chen Y, Pfeifer A (2017) Brown fat-derived exosomes: small vesicles with big impact. Cell Metab 25:759-760

13. Rahman MJ, Regn D, Bashratyan R, Dai YD (2014) Exosomes released by islet-derived mesenchymal stem cells trigger autoimmune responses in NOD mice. Diabetes 63:1008-1020

14. Pitt JM, Kroemer G, Zitvogel L (2016) Extracellular vesicles: masters of intercellular communication and potential clinical interventions. J Clin Invest 126:1139-1143

15. Garcia-Contreras M, Brooks RW, Boccuzzi L, Robbins PD, Ricordi C (2017) Exosomes as biomarkers and therapeutic tools for type 1 diabetes mellitus. Eur Rev Med Pharmacol Sci 21:2940-2956

16. Ruan Q, Wang T, Kameswaran V et al (2011) The microRNA-21 -PDCD4 axis prevents type 1 diabetes by blocking pancreatic $\beta$ cell death. Proc Natl Acad Sci 108:12030-12035

17. Roggli E, Britan A, Gattesco S et al (2010) Involvement of MicroRNAs in the cytotoxic effects exerted by proinflammatory cytokines on pancreatic $\beta$-Cells. Diabetes 59:978-986

18. Roggli E, Gattesco S, Caille D et al (2012) Changes in MicroRNA expression contribute to pancreatic $\beta$-cell dysfunction in prediabetic NOD mice. Diabetes 61:1742-1751

19. Osipova J, Fischer D-C, Dangwal S et al (2014) Diabetesassociated microRNAs in pediatric patients with type 1 diabetes mellitus: a cross-sectional cohort study. J Clin Endocrinol Metab 99:E1661-E1665

20. Seyhan AA, Nunez Lopez YO, Xie H et al (2016) Pancreasenriched miRNAs are altered in the circulation of subjects with diabetes: a pilot cross-sectional study. Sci Rep 6:31479

21. Hatanaka M, Anderson-Baucum E, Lakhter A et al (2017) Chronic high fat feeding restricts islet mRNA translation initiation independently of ER stress via DNA damage and p53 activation. Sci Rep 7: 3758

22. Scharfmann R, Pechberty S, Hazhouz Y et al (2014) Development of a conditionally immortalized human pancreatic $\beta$ cell line. J Clin Invest 124:2087-2098

23. Clancy JW, Sedgwick A, Rosse C et al (2015) Regulated delivery of molecular cargo to invasive tumour-derived microvesicles. Nat Commun 6:6919
24. Thery C, Amigorena S, Raposo G, Clayton A (2006) Isolation and characterization of exosomes from cell culture supernatants and biological fluids. Curr Protoc Cell Biol 3:3.22.1-3.22.29

25. Fisher MM, Watkins RA, Blum J et al (2015) Elevations in circulating methylated and unmethylated preproinsulin DNA in newonset type 1 diabetes. Diabetes 64:3867-3872

26. Prochazka M, Serreze DV, Frankel WN, Leiter EH (1992) NOR/Lt mice: MHC-matched diabetes-resistant control strain for NOD mice. Diabetes 41:98-106

27. Stull ND, Breite A, McCarthy R, Tersey SA, Mirmira RG (2012) Mouse islet of Langerhans isolation using a combination of purified collagenase and neutral protease. J Vis Exp 67:4137

28. Crescitelli R, Lasser C, Szabo TG et al (2013) Distinct RNA profiles in subpopulations of extracellular vesicles: apoptotic bodies, microvesicles and exosomes. J Extracell Vesicles. https://oi.org/ 10.3402/jev.v2i0.20677

29. Ha M, Kim VN (2014) Regulation of microRNA biogenesis. Nat Rev Mol Cell Biol 15:509-524

30. Snowhite IV, Allende G, Sosenko J, Pastori RL, Messinger Cayetano S, Pugliese A (2017) Association of serum microRNAs with islet autoimmunity, disease progression and metabolic impairment in relatives at risk of type 1 diabetes. Diabetologia 60:14091422

31. Erener S, Mojibian M, Fox JK, Denroche HC, Kieffer TJ (2013) Circulating miR-375 as a biomarker of $\beta$-cell death and diabetes in mice. Endocrinology 154:603-608

32. Song I, Roels S, Martens GA, Bouwens L (2017) Circulating microRNA-375 as biomarker of pancreatic beta cell death and protection of beta cell mass by cytoprotective compounds. PLoS One 12:e0186480

33. Guay C, Menoud V, Rome S, Regazzi R (2015) Horizontal transfer of exosomal microRNAs transduce apoptotic signals between pancreatic beta-cells. Cell Commun Signal 13:17

34. Sheng H, Hassanali S, Nugent $C$ et al (2011) Insulinoma-released exosomes or microparticles are immunostimulatory and can activate autoreactive $\mathrm{T}$ cells spontaneously developed in nonobese diabetic mice. J Immunol 187:1591-1600

35. Cantaluppi V, Biancone L, Figliolini F et al (2012) Microvesicles derived from endothelial progenitor cells enhance neoangiogenesis of human pancreatic islets. Cell Transplant 21:1305-1320

36. Palmisano G, Jensen SS, Le Bihan M-C et al (2012) Characterization of membrane-shed microvesicles from cytokinestimulated $\beta$-cells using proteomics strategies. Mol Cell Proteomics 11:230-243

37. Zhu Q, Kang J, Miao H et al (2014) Low-dose cytokine-induced neutral ceramidase secretion from INS-1 cells via exosomes and its anti-apoptotic effect. FEBS J 281:2861-2870

38. Vallabhajosyula P, Korutla L, Habertheuer A et al (2017) Tissuespecific exosome biomarkers for noninvasively monitoring immunologic rejection of transplanted tissue. J Clin Invest 127:13751391

39. Hasilo CP, Negi S, Allaeys I et al (2017) Presence of diabetes autoantigens in extracellular vesicles derived from human islets. Sci Rep 7:5000

40. Garcia-Contreras M, Shah SH, Tamayo A et al (2017) Plasmaderived exosome characterization reveals a distinct microRNA signature in long duration type 1 diabetes. Sci Rep 7:5998

41. Turchinovich A, Samatov TR, Tonevitsky AG, Burwinkel B (2013) Circulating miRNAs: cell-cell communication function? Front Genet 4:119

42. Erener S, Marwaha A, Tan R, Panagiotopoulos C, Kieffer TJ (2017) Profiling of circulating microRNAs in children with recent onset of type 1 diabetes. JCI Insight 2:e89656

43. Nielsen LB, Wang C, Sørensen K et al (2012) Circulating levels of microRNA from children with newly diagnosed type 1 diabetes and healthy controls: evidence that miR-25 associates to residual beta- 
cell function and glycaemic control during disease progression. Exp Diabetes Res 2012:896362

44. Akirav EM, Lebastchi J, Galvan EM et al (2011) Detection of $\beta$ cell death in diabetes using differentially methylated circulating DNA. Proc Natl Acad Sci U S A 108:19018-19023
45. Pesenacker AM, Wang AY, Singh A et al (2016) A regulatory T cell gene signature is a specific and sensitive biomarker to identify children with new-onset type 1 diabetes. Diabetes 65:1031

46. Krichevsky AM, Gabriely G (2009) miR-21: a small multi-faceted RNA. J Cell Mol Med 13:39-53 